\title{
Pd-Sensitized Single Vanadium Oxide Nanowires: Highly Responsive Hydrogen Sensing Based on the Metal-Insulator Transition
}

\author{
Jeong Min Baik, ${ }^{\dagger, \neq}$ Myung Hwa Kim, ${ }^{\dagger, \S}$ Christopher Larson, Cafer T. Yavuz, \\ Galen D. Stucky, Alec M. Wodtke, ${ }^{,}$and Martin Moskovits* \\ Department of Chemistry \& Biochemistry, University of California, \\ Santa Barbara, California 93106
}

Received June 23, 2009; Revised Manuscript Received October 18, 2009

\begin{abstract}
Exceptionally sensitive hydrogen sensors were produced using Pd-nanoparticle-decorated, single vanadium dioxide nanowires. The highsensitivity arises from the large downward shift in the insulator to metal transition temperature following the adsorption on and incorporation of atomic hydrogen, produced by dissociative chemisorption on $\mathrm{Pd}$, in the $\mathrm{VO}_{2}$, producing $\sim 1000$-fold current increases. During a rapid initial process, the insulator to metal transition temperature is decreased by $>10^{\circ} \mathrm{C}$ even when exposed to trace amounts of hydrogen gas. Subsequently, hydrogen continues to diffuse into the $\mathrm{VO}_{2}$ for several hours before saturation is achieved with only a modest change in the insulator to metal transition temperature but with a significant increase in the conductivity. The two time scales over which H-related processes occur in $\mathrm{VO}_{2}$ likely signal the involvement of two distinct mechanisms influencing the electronic structure of the material one of which involves electron-phonon coupling pursuant to the modification of the vibrational normal modes of the solid by the introduction of $\mathrm{H}$ as an impurity.
\end{abstract}

One-dimensional (1-D) nanostructures of metal-oxide semiconductors are currently the subject of intense research both in order to discover fundamental science at the nanoscale as well as for their potential as sensing, catalytic, and other electronic applications. ${ }^{1-9}$ One paradigm for improving the selectivity and high-sensitivity of nanowire-based gas sensors is to functionalize their surfaces with metal nanoparticles thereby refining their performance through the specificity of the catalysis taking place on the metal. ${ }^{10,11}$

Vanadium dioxide $\left(\mathrm{VO}_{2}\right)$ is an interesting material on account of its easily accessible and sharp Mott metal-insulator transition (MIT) at $\sim 68{ }^{\circ} \mathrm{C}$ in the bulk. ${ }^{12-14}$ In this Letter, we report an unusually large shift in the metal-insulator transition of $\mathrm{VO}_{2}$ nanowires by hydrogen atoms adsorbed on its surface and incorporated into its lattice following catalytic dissociation of $\mathrm{H}_{2}$ on and in $\mathrm{Pd}$ nanoparticles deposited on the nanowire's surface. This catalytic process produces a large dose of atomic hydrogen at the nanowire's surface, rapidly lowering its insulator to metal transition

* To whom all correspondence should be addressed: mmoskovits@ ltsc.ucsb.edu and wodtke@chem.ucsb.edu.

$\uparrow$ These authors contributed equally to this work.

* Current address: School of Advanced Materials and Systems Engineering, Kumoh National Institute of Technology, Gumi, Gyungbuk, 730-701, Korea.

${ }^{\S}$ Current address: Department of Chemistry and Nano Science, Ewha Womans University, Seoul, 120-750, Korea. temperature by over $10{ }^{\circ} \mathrm{C}$, an effect that can potentially be exploited to create inordinately sensitive hydrogen sensors and may also point to a potentially fruitful paradigm for developing hydrogen storage materials. Recently Strelcov et al. proposed using the Mott transition in $\mathrm{VO}_{2}$ nanowires as an all-purpose temperature sensor. ${ }^{15}$ However, in the absence of functionalization, their system shows no specificity to $\mathrm{H}$ nor is the depression in Mott transition temperature as large as what we report here.

Single-crystal vanadium oxide nanowires were grown by atmospheric pressure, physical vapor deposition as described previously. ${ }^{16}$ Briefly, $0.1 \mathrm{~g}$ of fine mesh $\mathrm{VO}_{2}$ (99.9\%, Aldrich) powder was placed at the center of a $10 \mathrm{~cm}$ long quartz boat without further purification. A precleaned $\mathrm{SiO}_{2} /$ Si substrate was located in the quartz boat approximately 5 $\mathrm{mm}$ from the $\mathrm{VO}_{2}$. The boat was then placed at the center of a quartz tube furnace and high-purity $\mathrm{He}$ carrier gas $(99.999 \%)$ was flowed through the furnace at $300 \mathrm{sccm}$. Experiments with air rather than $\mathrm{He}$ as the carrier gas were also conducted. The furnace temperature was increased to approximately $650{ }^{\circ} \mathrm{C}$. Sample growth proceeded for $2 \mathrm{~h}$ following which the samples were allowed to cool to room temperature in flowing $\mathrm{He}$ and the nanowire-covered substrate was removed from the furnace. Field-emission scanning electron microscopy (FEI Sirion XL30-FEG) was used 


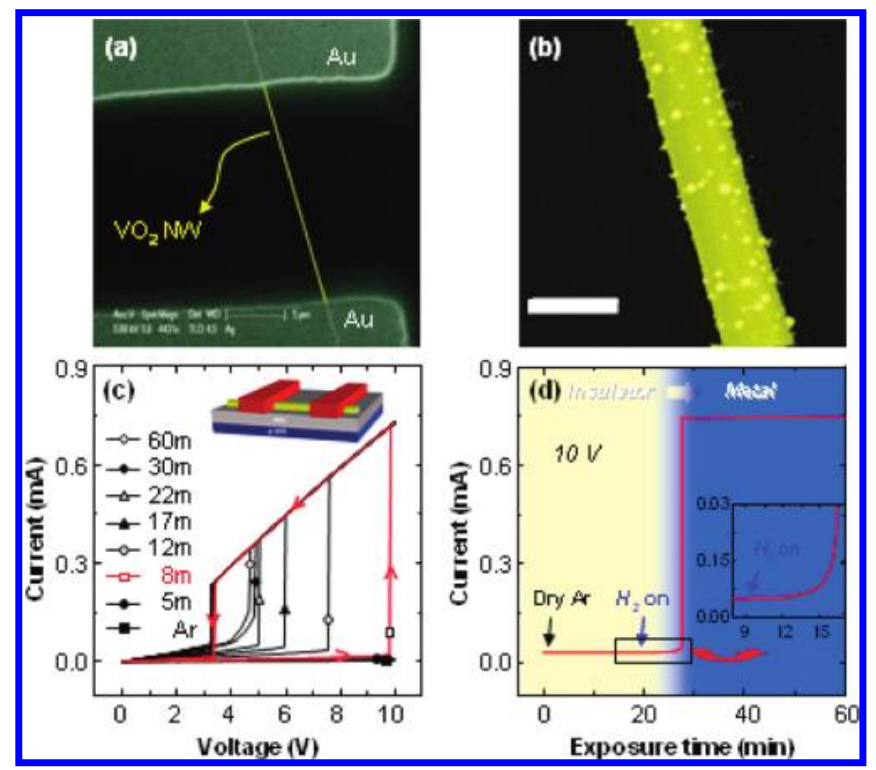

Figure 1. (a) Scanning electron microscopy (SEM) image of an individual $\mathrm{VO}_{2}$ nanowire device configured with appropriate Ohmic contacts for electrical measurements in a gaseous atmosphere. (b) SEM image of a $\mathrm{Pd}$-decorated $\mathrm{VO}_{2}$ nanowire. The Pd particles, $5-22 \mathrm{~nm}$ in diameter, are noncontinuous and cover the surface of the nanowire uniformly (scale bar, $200 \mathrm{~nm}$ ). (c) $I-V$ curves obtained at $50{ }^{\circ} \mathrm{C}$ for $\mathrm{Pd}$-decorated $\mathrm{VO}_{2}$ nanowire after various exposure times to hydrogen gas $(5 \mathrm{sccm})$, added to the background argon stream $(10 \mathrm{sccm})$. (d) The change in current for a Pd-decorated $\mathrm{VO}_{2}$ nanowire biased at $10 \mathrm{~V}$ as a function of time of exposure to hydrogen gas. Initially the current increases gradually with hydrogen exposure time and then at $\sim 7 \mathrm{~min}$ increases dramatically by $\sim 3$ orders of magnitude $\left(5 \times 10^{-6} \mathrm{~A} \rightarrow 6 \times 10^{-3} \mathrm{~A}\right)$ in the absence of the series resistor.

to image the $\mathrm{VO}_{2}$ nanowires. Electrical contacts were deposited onto individual $\mathrm{VO}_{2}$ nanowires residing on the $\mathrm{SiO}_{2} / \mathrm{Si}$ growth substrates. Ti/Au (10/400 nm) electrodes were fabricated by conventional e-beam metal vapor deposition through a shadow mask. To minimize contamination, no wet processes (such as photoresist-based lithography) were employed at any stage of the device fabrication. Current-voltage measurements were carried out on isolated individual nanowires in a custom-built chamber in which the temperature and gas composition could be varied. Ambient gas composition was controlled by mass flow meters. All gas partial pressures were in the range 1-100 mTorr. Samples were annealed in nitrogen for $1 \mathrm{~min}$ at $200{ }^{\circ} \mathrm{C}$ to improve the Ohmic contact between the nanowire and metallic micropads. Finally, a $\sim 0.6 \mathrm{~nm}$ thick (mass thickness) $\mathrm{Pd}$ film, which subsequently aggregated into well-spaced $\mathrm{Pd}$ nanoparticles, was deposited over the devices using electron beam evaporation at a base pressure of $2.0 \times 10^{-6}$ Torr.

All $I-V$ measurements were carried out in a two-terminal configuration in series with a $10 \mathrm{k} \Omega$ resistor and at ambient temperatures of $45,50,55$, and $60{ }^{\circ} \mathrm{C}$, with and without $\mathrm{Pd}$ nanoparticles decorating the surface of the nanowires. Figure $1 \mathrm{c}$ shows a typical set of results obtained at $50{ }^{\circ} \mathrm{C}$ for a $\mathrm{Pd}$ decorated $\mathrm{VO}_{2}$ nanowire exposed to hydrogen flowing at 5 sccm added for various lengths of time to a $10 \mathrm{sccm}$ background argon stream. The hysteresis in the $I-V$ measurements result from the MIT-induced by Ohmic heating

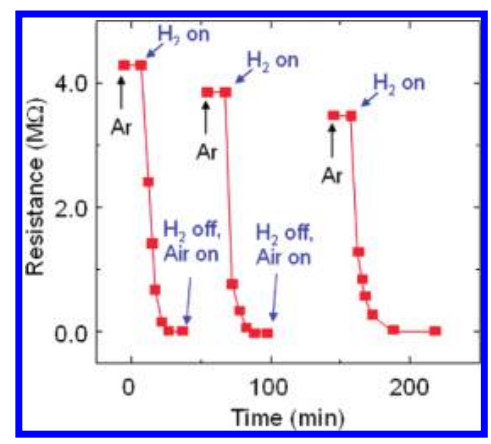

Figure 2. Rate of return of the resistance of a Pd-decorated $\mathrm{VO}_{2}$ nanowire biased at $5 \mathrm{~V}$ toward its initial value when recovery in air was carried out at $100{ }^{\circ} \mathrm{C}$, reducing the recovery time to 30 $\min$. The data plotted were obtained in a continuous experiment with the $\mathrm{H}_{2}$ flow turned on an off at the points shown.

of the nanowire. ${ }^{15,16}$ Initiating the hydrogen flow across a Pd-decorated $\mathrm{VO}_{2}$ nanowire biased at $10 \mathrm{~V}$ causes the current to increase steadily (Figure 1d) and then increase approximately 1000-fold after $\sim 10 \mathrm{~min}$ of $\mathrm{H}_{2}$ exposure at ambient temperatures in the range $45-55^{\circ} \mathrm{C}$, or after less than 5 min when the ambient temperature was $60{ }^{\circ} \mathrm{C}$, signaling the occurrence of the insulator to metal transition. The result of a similar experiment run in air is shown in Figure S2 in the Supporting Information. In contrast, turning on the hydrogen flow in the absence of the Pd nanoparticles produced $\leq 10 \%$ changes (Figure S2 in Supporting Information). We ascribe this to the presence of large quantities of $\mathrm{H}$ atoms on the nanowire's surface produced by catalytic dissociation of diatomic hydrogen on and in the Pd nanoparticles. The $\mathrm{H}$ atoms "spill over" the nanowire's surface ${ }^{17,18}$ and then diffuse into the vanadium oxide.

On termination of the hydrogen exposure after $60 \mathrm{~min}$, the recovery of the nanowire's conductance toward its initial value was found to be very slow. Even after $24 \mathrm{~h}$ at room temperature in vacuum $\left(<10^{-5}\right.$ Torr $)$ the conductance of the $\mathrm{VO}_{2}$ nanowire did not change significantly. Upon introducing air into the chamber, the device current began to decrease immediately (likely due to the catalytic oxidation of the $\mathrm{H}$ by the Pd to form water) but nevertheless required over $4 \mathrm{~h}$ at $50{ }^{\circ} \mathrm{C}, 30 \mathrm{~min}$ at $80{ }^{\circ} \mathrm{C}$, and $5 \mathrm{~min}$ at $100{ }^{\circ} \mathrm{C}$ (Figure 2 and Figure S3 in Supporting Information) for the current to revert to its initial value. Catalytic processes occurring on metallic nanoparticles deposited on metal-oxide semiconductor nanowires other than vanadium oxide have been reported previously. ${ }^{8,10,17,18}$

The electrical conductance of the $\mathrm{VO}_{2}$ nanowire is found to change over two time scales: a fast process taking place over 1-5 min, depending on the ambient temperature, and a slow process which takes many hours to reach a steady value. For example, with a $1 \mathrm{~V}$ bias, conductance steady state is reached after $\sim 18 \mathrm{~h}$ of $\mathrm{H}_{2}$ exposure (Figure 3a). We find, however, that the time constant for the initial fast process could be significantly reduced by increasing the Pd coverage on the surface (Figure S4 in Supporting Information). For example, for Pd coverage corresponding to a 1 $\mathrm{nm}$ mass thickness, the response time is reduced from minutes to tens of seconds. This acceleration in response 


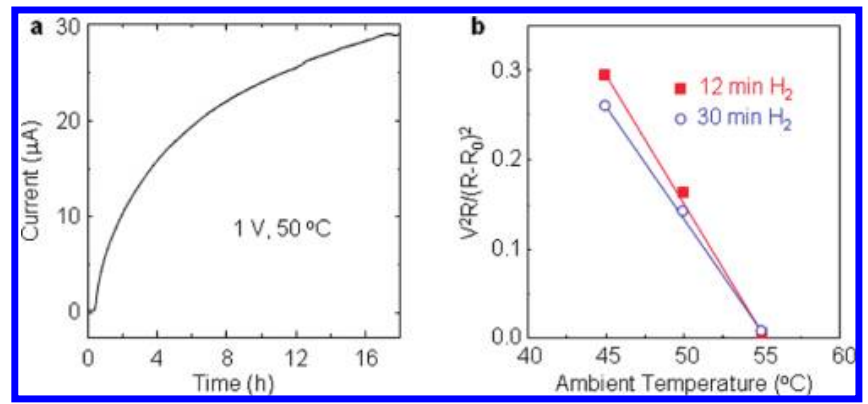

Figure 3. (a) The change in the current of a Pd-decorated $\mathrm{VO}_{2}$ nanowire biased at $1 \mathrm{~V}$ with exposure time to hydrogen gas. Saturation occurs after $18 \mathrm{~h}$. (b) Plots of $V_{\mathrm{M}}{ }^{12} R_{\mathrm{cM}} /\left(R_{0}-R_{\mathrm{cM}}\right)^{2}$ as a function of the ambient temperature, $T_{0}$. The intercept implies that the metal-insulator transition temperature, $T_{\mathrm{M}}$, is reduced to $55-56{ }^{\circ} \mathrm{C}$ after 12 min of exposure to $\mathrm{H}_{2}$ under our experimental conditions.

time is likely due to the increased number of sources of atomic hydrogen on the nanowire's surface and, therefore, the increased rate of atomic $\mathrm{H}$ incorporation in the surface region of the $\mathrm{VO}_{2}$.

Referring to Figure $2 b$, we note that as the bias voltage is ramped upward, the voltage at which the insulator to metal transition occurs moves to lower voltage values with increasing hydrogen exposure. Similar behavior is seen for the reverse metal to insulator transition as the bias voltage is ramped downward. More noteworthy is the observation that as the ambient temperature is increased the voltage at which the reverse (i.e., metal to insulator) transition takes place trends significantly to lower voltage values, so that at an ambient temperature of $60{ }^{\circ} \mathrm{C}$ the $\mathrm{Pd}$-decorated nanowire exists only in its metallic phase after less than $5 \mathrm{~min}$ of $\mathrm{H}_{2}$ exposure (Figure S5 in Supporting Information), suggesting that the metal-insulator transition temperature had been depressed below $60{ }^{\circ} \mathrm{C}$.

Previously we showed that one could obtain a quantitative estimate of the metal-insulator transition temperature of a $\mathrm{VO}_{2}$ nanowire from the expressions

$$
\begin{aligned}
V_{\mathrm{M}}{ }^{\downarrow} & =\left[\frac{k\left(T_{\mathrm{M}}-T_{0}\right)\left(R_{0}+R_{\mathrm{cD}}\right)^{2}}{R_{\mathrm{cD}}}\right]^{1 / 2} \\
V_{\mathrm{M}}{ }^{\downarrow} & =\left[\frac{k\left(T_{\mathrm{M}}-T_{0}\right)\left(R_{0}+R_{\mathrm{cM}}\right)^{2}}{R_{\mathrm{cM}}}\right]^{1 / 2}
\end{aligned}
$$

in which $V_{\mathrm{M}}{ }^{\uparrow}$ and $V_{\mathrm{M}}{ }^{\dagger}$ are the bias voltages at which the MIT occurs when sweeping the bias voltage, respectively, from lower to higher and higher to lower voltages, $T_{\mathrm{M}}$ and $T_{0}$, are the metal-insulator transition and ambient temperatures, respectively, $R_{0}, R_{\mathrm{cD}}$ and $R_{\mathrm{cM}}$ are, respectively, the resistance in series with the $\mathrm{VO}_{2}$ nanowire (which may include the contact resistance), the resistance of the nanowire in its insulator phase, and its resistance in the metallic phase, and $k$ is a collection of constants proportional to the heat conductance of the nanowire. ${ }^{16}$

When $R_{0}<R_{\mathrm{cD}}$ (which is always so for the nanowires we measured) eq 1 can take the simpler approximate form

$$
V_{\mathrm{M}}{ }^{\dagger}=\left[k\left(T_{\mathrm{M}}-T_{0}\right) R_{\mathrm{cD}}\right]^{1 / 2}
$$

Rearranging eq $1^{\prime}$, one obtains the expression

$$
\frac{V_{\mathrm{M}}{ }^{12} R_{\mathrm{cM}}}{\left(R_{0}-R_{\mathrm{cM}}\right)^{2}}=k\left(T_{\mathrm{M}}-T_{0}\right)
$$

Values of the function represented by the left-hand side of eq 2 were calculated from the measured values of $V_{\mathrm{M}}{ }^{\downarrow}$ and plotted as a function of $T_{0}$ (Figure $3 \mathrm{~b}$ ). Assuming the metal-insulator transition for the hydrogen-free nanowire occurs at $68{ }^{\circ} \mathrm{C}$ (its bulk value), an estimate of $T_{\mathrm{M}}$ induced by hydrogen exposure can be obtained from the $x$-axis intercept, that is, where $T_{\mathrm{M}}=T_{0}$ ). This is found to be $55-56{ }^{\circ} \mathrm{C}$ after $12 \mathrm{~min}$ of exposure to $\mathrm{H}_{2}$ under our experimental conditions. Interestingly, one obtains approximately the same value of $T_{\mathrm{M}}$ from the data obtained after $30 \mathrm{~min}$ of hydrogen exposure. This suggests that the continued downward shift of the metal-insulator transition voltage observed with continued hydrogen exposure and incorporation beyond $12 \mathrm{~min}$ (under ambient temperatures in the range $45-55^{\circ} \mathrm{C}$ ) when the voltage across the nanowire is ramped upward (Figure 2b), is due largely to the dramatic, hydrogen-induced decrease in the nanowire's resistivity (approximately in accordance with eq $1^{\prime \prime}$ ), rather than to further decreases in the metal-insulator transition temperature. This might signal the involvement of two mechanisms relating to the electronic structure of $\mathrm{VO}_{2}$ : one involving electron-phonon coupling influenced by the introduction of $\mathrm{H}$ impurities whose masses alter the vibrational normal modes of the material, the second due to the modification of the material's band structure accompanying $\mathrm{H}$ infusion due presumably to electrons released into its conduction band by interstitial $\mathrm{H}$. The competing roles of electron-phonon and electron-electron coupling to the structural and electronic properties of $\mathrm{VO}_{2}$ are an area of active interest. ${ }^{19-21}$

Preliminary Raman studies on Pd-decorated $\mathrm{VO}_{2}$ nanowires measured as a function of ambient temperature and subsequent to dosing with hydrogen showed two Raman lines $\left(191,223 \mathrm{~cm}^{-1}\right)$ present at low temperatures characteristic of the monoclinic phase which disappear when the metal-insulator transition temperature is reached (Supporting Information, Figure S6). Dosing with hydrogen was found to depress the temperature at which those Raman lines disappeared by a temperature interval consistent with what we report above.

Dissociative chemisorption of hydrogen on Pd surfaces followed by the subsequent incorporation as atomic hydrogen in the Pd crystal to form a solid hydride is a well-known process. ${ }^{18}$ The overall rate of formation of the bulk $\mathrm{Pd}-\mathrm{H}$ phase at $50{ }^{\circ} \mathrm{C}$ is observed to be $\sim 2.3$ higher for hydrogen as compared to deuterium. ${ }^{22}$ The contributing steps in that reaction include dissociative chemisorption, dissolution into the surface region of the metal followed by transport into the bulk. Although deuterium atoms are known to diffuse more rapidly through Pd than hydrogen atoms, the overall mass flow of hydrogen through $\mathrm{Pd}$ is greater, because $\mathrm{H}$ dissolves in palladium preferentially to $\mathrm{D}$, and the higher concentration gradients thus built up outweigh the higher mobility of individual deuterium atoms. Nevertheless, the magnitudes of the rates of hydrogen or deuterium atom incorporation into $\mathrm{Pd}$ are of the same order of magnitude as their diffusion rates.

For Pd-nanoparticle-decorated $\mathrm{VO}_{2}$ nanowires, the situation is potentially more complex since, in addition to $\mathrm{H}$ atom formation and its interaction with the $\mathrm{Pd}$, one has processes 


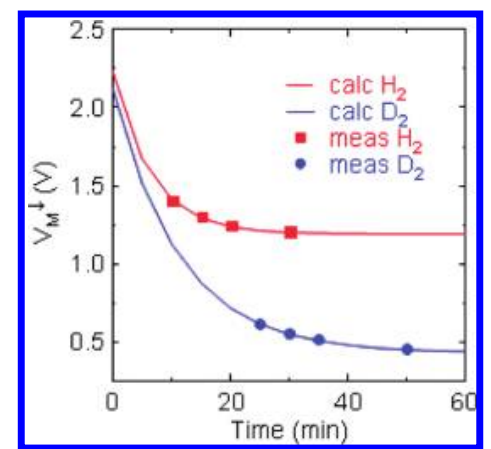

Figure 4. Experimental (points) values of $V_{\mathrm{M}} \downarrow$ as a function of exposure time to hydrogen or deuterium gases fitted to the function given by using eq 3 (solid curves). The fit returned the following parameters: for hydrogen: $V_{\infty}=1.2 \mathrm{~V}, A=1.04 \mathrm{~V}$, and $\gamma=0.0026$ $\mathrm{s}^{-1}$; for deuterium: $V_{\infty}=0.43 \mathrm{~V}, A=1.7 \mathrm{~V}$, and $\gamma=0.0015 \mathrm{~s}^{-1}$.

such as $\mathrm{H}$ atom migration onto the oxide's surface and its penetration into the oxide's surface and bulk regions. We investigated the isotope effect on these processes by measuring the electrical response of the Pd-decorated $\mathrm{VO}_{2}$ nanowire when exposed alternately to hydrogen or deuterium gas under $5 \mathrm{~V}$ bias. Figure 4 shows a plot of $V_{\mathrm{M}}^{\downarrow}$ (cf. Figure $2 \mathrm{~b}$ ) as a function of exposure time to hydrogen or deuterium gases. The kinetic data shown in Figure 4 obey an equation of the form

$$
V_{\mathrm{M}}{ }^{\downarrow}=V_{\infty}+A \exp (-\gamma t)
$$

The values of $V_{\infty}$ and $V_{\infty}+A$ are the final and initial values of $V_{\mathrm{M}}{ }^{\downarrow}$, respectively, and $\gamma$ is a decay rate, i.e., a measure of the rate-determining process in the reaction/diffusion scheme that governs the incorporation of hydrogen in the Pddecorated $\mathrm{VO}_{2}$ nanowire. Fitting eq 3 to the measured data produces an excellent fit (Figure 4) and the following parameter set. For hydrogen: $V_{\infty}=1.2 \mathrm{~V}, A=1.04 \mathrm{~V}$, and $\gamma=0.0026 \mathrm{~s}^{-1}$; for deuterium: $V_{\infty}=0.43 \mathrm{~V}, A=1.7 \mathrm{~V}$, and $\gamma=0.0015 \mathrm{~s}^{-1}$. Note that the value of $\left(V_{\infty}\right)^{2}$ for $\mathrm{D}$ is lower than that for $\mathrm{H}$ by a factor of $\sim 7$, which implies that the dissolution of $\mathrm{D}$ atoms in $\mathrm{VO}_{2}$ brings about a greater depression in the insulator to metal transition temperature than does $\mathrm{H}$. This fact might also imply the connection between the material's phonon structure and the dynamics of its Mott transition. (We showed above and previously ${ }^{16}$ that $\left(V_{\infty}\right)^{2}$, that is, the value of $V_{\mathrm{M}}{ }^{12}$ at $t=\infty$ is a measure of the difference between the metal-insulator transition temperature and the ambient temperature after the system reaches saturation with hydrogen.) By contrast, $\mathrm{H}$ seems to reach a steady-state value faster than does $\mathrm{D}$, with an effective rate constant $(\gamma)$ ratio $\sim 1.7$. It is gratifying to note that although the two sets of data were fit independently and the values of $V_{\infty}$ and $A$ differ significantly for $\mathrm{D}$ and $\mathrm{H}$, the quantities $V_{\infty}$ $+A$ come out to be approximately equal for the two isotopes (2.2 V for $\mathrm{H}_{2}$ and $2.1 \mathrm{~V}$ for $\mathrm{D}_{2}$ ), as one would expect for initial values prior to the nanowire's exposure to either $\mathrm{H}_{2}$ or $\mathrm{D}_{2}$.

Which of the two processes, production of the $\mathrm{Pd} / \mathrm{H}$ phase or diffusion of hydrogen into the $\mathrm{VO}_{2}$, is the rate-determining process may be obtained as follows. An order of magnitude rate for a diffusional process can be determined using the expression $^{18}$

$$
\gamma_{\mathrm{e}}^{-1}=\tau=\left(\frac{L^{2}}{D(T)}\right)
$$

In which $\tau$ is the diffusion time whose inverse, $\gamma_{\mathrm{e}}$, functions as an effective diffusion rate constant, and $L$ is the diffusion length. The temperature-dependent diffusion coefficient is given by

$$
D(T)=D_{0} \exp \left(\frac{-E_{\mathrm{a}}}{k_{\mathrm{B}} T}\right)
$$

where $E_{\mathrm{a}}$ and $k_{\mathrm{B}}$ are the activation energy and the Boltzmann constant, respectively. Using published values for $D_{0}$ and $E_{\mathrm{a}}$, one calculates the diffusion coefficients for atomic hydrogen and deuterium through $\mathrm{Pd}$ at $50{ }^{\circ} \mathrm{C}$ to be $7.45 \times$ $10^{-7} \mathrm{~cm}^{2} / \mathrm{s}$ and $1.03 \times 10^{-6} \mathrm{~cm}^{2} / \mathrm{s}$, respectively. ${ }^{23}$

Substituting these values for the diffusion constant into eq 4 and taking $L$ to be $10 \mathrm{~nm}$, the mean diameter of the deposited Pd particles, one calculates the following values of $\gamma_{\mathrm{e}}: 7.5 \times 10^{5}$ and $1.0 \times 10^{6} \mathrm{~s}^{-1}$, respectively, for $\mathrm{H}$ and $\mathrm{D}$ diffusion into $\mathrm{Pd}$. These rates are some 8 orders of magnitude larger than what we observed, suggesting that penetration into the $\mathrm{VO}_{2}$ nanowire is the rate-determining process and that H/D diffusion through the Pd is essentially complete when those involving the oxide are just beginning. The observed isotope effect is also reversed from that expected for transport of $\mathrm{H}$ through $\mathrm{Pd}$ : the diffusion rate for $\mathrm{H}$ in $\mathrm{VO}_{2}$ is faster than that for $\mathrm{D}$, while in $\mathrm{Pd}$ the opposite is true. Our observation is, however, consistent with previous reports on hydrogen diffusion into $\mathrm{VO}_{2}$ films. ${ }^{24,25}$

Summarizing, $\mathrm{Pd}$-decorated $\mathrm{VO}_{2}$ nanowire sensors show extraordinary sensitivity toward hydrogen. This comes about as a result of the rapid dissociation of the diatomic hydrogen on the Pd nanoparticles followed by the migration of the atomic hydrogen over the surface of the $\mathrm{VO}_{2}$ nanowire and its diffusion into the vanadia lattice, causing a significant downward shift in the metal-insulator transition temperature. By biasing the nanowire judiciously so that its temperature is just below the metal-insulator transition temperature, even trace amounts of hydrogen induce the metal-insulator transition, producing an almost 3 order of magnitude increase in the current through the nanowire. Although a sensor based on this process operates only near the metal-insulator transition temperature, one can operate the device at essentially any ambient temperature below ca. $60{ }^{\circ} \mathrm{C}$ by resistively heating the nanowire judiciously so as to set the local temperature of the nanowire close to the insulator to metal transition temperature regardless of the temperature of the ambient. Although the study was carried out to illustrate the principle rather than to probe the ultimate sensitivity of this device as a sensor, the metal-insulator transition temperature was depressed over $10{ }^{\circ} \mathrm{C}$ with hydrogen partial pressures $<5 \times 10^{-6} \mathrm{~atm}$. Since, significant current changes can be measured with temperature depressions $\sim 0.1{ }^{\circ} \mathrm{C}$, a hydrogen sensor with a parts-per-billion sensitivity should be possible based on this phenomenon.

The large change in the Mott transition temperature of $\mathrm{VO}_{2}$ likely results from the formation of hydrogen interstitials or to the formation of adsorbed $\mathrm{OH}$, both of which act as electron donors increasing the carrier density in the conduction band. The carrier density increase with increasing $\mathrm{H}$ 
atom incorporation is observed as a steady and significant conductance increase of the nanowire while in its insulator phase. This initial process, which is complete in a few minutes, causes a shift in insulator to metal transition temperature decrease of over $10{ }^{\circ} \mathrm{C}$. Following this initial process, which is largely confined to the surface region, hydrogen continues to penetrate the $\mathrm{VO}_{2}$ nanowire over several hours, continually increasing its conductance while in its insulator phase, without further decreasing its metal-insulator transition temperature significantly. This effect is also likely due to the formation of filled donor states. $^{26}$

Acknowledgment. This work was supported by the Institute for Collaborative Biotechnologies through Grant DAAD19-03-D- 0004 from the U.S. Army Research Office and made extensive use of the MRL Central Facilities at UCSB supported by the National Science Foundation under Award Nos. DMR-0080034 and DMR-0216466 for the HRTEM/STEM microscopy. We gratefully acknowledge the financial support from the Partnership for International Research and Education-for Electronic Chemistry and Catalysis at Interfaces-NSF Grant Number OISE-0530268.

Supporting Information Available: Figures showing SEM images of $\mathrm{VO}_{2}$ nanowires, hydrogen response of $\mathrm{Pd}-\mathrm{VO}_{2}$ nanowires in air and bare $\mathrm{VO}_{2}$ nanowires in argon, reversibility with ambient temperature, hydrogen response time with $\mathrm{Pd}$ thickness, hydrogen response at $60{ }^{\circ} \mathrm{C}$, and Raman spectra as a function of ambient temperature. This material is available free of charge via the Internet at http:// pubs.acs.org.

\section{References}

(1) Cui, Y.; Wei, Q. Q.; Park, H. K.; Lieber, C. M. Science 2001, 293, $1289-1292$.

(2) Law, M.; Kind, L. H.; Messer, B.; Kim, F.; Yang, P. D. Angew. Chem. Int. Ed. 2002, 41, 2405-2408.
(3) Wu, J.; Gu, Q.; Guiton, B. S.; de Leon, N. P.; Ouyang, L.; Park, H. Nano Lett. 2006, 6, 2313-2317.

(4) Kolmakov, A.; Zhang, Y.; Cheng, G.; Moskovits, M. Adv. Mater. 2003, 15, 997-1000.

(5) Arnold, M. S.; Avouris, P.; Pan, Z. W.; Wang, Z. L. J. Phvs. Chem. B 2003, 107, 659-663.

(6) Li, C.; Zhang, D. H.; Liu, X. L.; Han, S.; Tang, T.; Han, J.; Zhou, C. W. Appl. Phys. Lett. 2003, 82, 1613-1615.

(7) Wang, Y. L.; Jiang, X. C.; Xia, Y. N. J. Am. Chem. Soc. 2003, 125, 16176-16177.

(8) Kolmakov, A.; Klenov, D. O.; Lilach, Y.; Stemmer, S.; Moskovits, M. Nano Lett. 2005, 5, 667-673.

(9) Li, Z.; Zhang, H.; Zheng, W.; Wang, W.; Huang, H.; Wang, C.; MacDiarmid, A. G.; Wei, Y. J. Am. Chem. Soc. 2008, 130, 50365037.

(10) Wang, H. T.; Kang, B. S.; Ren, F.; Tien, L. C.; Sadik, P. W.; Norton, D. P.; Pearton, S. J.; Lin, J. Appl. Phvs. Lett. 2005, 86, 243503.

(11) Chen, X. H.; Moskovits, M. Nano Lett. 2007, 7, 807-812.

(12) Berglund, C. N.; Guggenheim, H. J. Phys. Rev. 1969, 185, 10221033.

(13) Eyert, V. Ann. Phvs. 2002, 11, 650-704.

(14) Biermann, S.; Poteryaev, A.; Lichtenstein, A. I.; Georges, A. Phvs. Rev. Lett. 2005, 94, 026404

(15) Strelcov, E.; Lilach, Y.; Kolmakov, A. Nano Lett. 2009, 9, 23222326.

(16) Baik, J. M.; Kim, M. H.; Larson, C.; Wodtke, A. M.; Moskovits, M. J. Phvs. Chem. C 2008, 112, 13328-13331.

(17) Khoobiar, S. J. Phys. Chem. 1964, 68, 411-412.

(18) Boudart, M.; Vannice, M. A.; Benson, J. E. J. Phvs. Chem. 1969, 64, 171-177.

(19) Okazaki, K.; Wadati, H.; Fujimori, A.; Onoda, M.; Muraoka, Y.; Hiroi, Z. Phvs. Rev. B 2004, 69, 165104.

(20) Okazaki, K.; Sugai, S.; Muraoka, Y.; Hiroi, Z. Phvs. Rev. B 2006, $73,165116$.

(21) Ruzmetov, D.; Zawilski, K. T.; Senanayake, S. D.; Narayanamurti, V.; Ramanathan, S. J. Phvs.: Condens. Matter. 2008, 20, 465204.

(22) Kong, J.; Chapline, M. G.; Dai, H. J. Adv. Mater. 2001, 13, 13841386.

(23) Wicke, E.; Nernst, G. H. Phys. Chem. 1964, 68, 224-235.

(24) Bermudez, V. M.; Williams, R. T.; Long, J. P.; Reed, R. K.; Klein, R. H. Phys. Rev. B 1992, 45, 9266-9271.

(25) Goodenough, J. B. J. Solid State Chem. 1971, 3, 490-500.

(26) King, P. D. C.; Lichti, R. L.; Celebi, Y. G.; Gil, J. M.; Vilão, R. C.; Alberto, H. V.; Duarte, J. P.; Payne, D. J.; Egdell, R. G.; McKenzie, I.; McConville, C. F.; Cox, S. F. J.; Veal, T. D. Phvs. Rev. B 2009, 80, 081201(R).

NL902020T 